\title{
Functional Characterization of Arabidopsis thaliana WRKY39 in Heat Stress
}

\author{
Shujia $\mathrm{Li}^{1,2}$, Xiang Zhou ${ }^{2}$, Ligang Chen ${ }^{2}$, Weidong Huang ${ }^{1, \star}$, and Diqiu $\mathrm{Yu}^{2, \star}$
}

\begin{abstract}
Arabidopsis thaliana WRKY39, a transcription factor that is induced by heat stress, is a member of the group II WRKY proteins and responds to both abiotic and biotic stress. Heat-treated seeds and plants of WRKY39 knock-down mutants had increased susceptibility to heat stress, showing reduced germination, decreased survival, and elevated electrolyte leakage compared with wild-type plants. In contrast, WRKY39 over-expressing plants exhibited enhanced thermotolerance compared with wild-type plants. RT-PCR and qRT-PCR analysis of wrky39 mutants and WRKY39 over-expressing plants identified putative genes regulated by WRKY39. Consistent with a role for WRKY39 in heat tolerance, the expression levels of salicylic acid (SA)regulated $P R 1$ and SA-related MBF1C genes were downregulated in wrky 39 mutants. In contrast, over-expression of WRKY39 increased the expression of PR1 and MBF1c. The WRKY39 transcript was induced in response to treatment with SA or methyljasmonate. Analysis of heat stressinduced WRKY39 in defense signaling mutants, including coi1, ein2, and sid2, further indicated that WRKY39 was positively co-regulated by the SA and jasmonate (JA) signaling pathways. Together, these findings reveal that heat stress-induced WRKY39 positively regulates the cooperation between the SA- and JA-activated signaling pathways that mediate responses to heat stress.
\end{abstract}

\section{INTRODUCTION}

Extreme temperature is an adverse environmental stress that severely impairs plant growth and development (Guy, 1999). Plants have a natural capacity to ameliorate the effects of heat shock (HS) (Hong and Vierling, 2000). Previous studies have shown that HS transcription factors (Hsfs) play an important role in thermotolerance in plants and other organisms by regulating HS proteins (Hsps) (Baniwal et al., 2004; Mishra et al., 2002; Panchuk et al., 2002; von Koskull-Döring et al., 2007). However, plants with mutations disrupting abscisic acid (ABA), salicylic acid (SA), hydrogen peroxide, ethylene (ET), or calcium signaling, which appear to accumulate similar Hsp101 and other Hsps to wild-type plants, displayed thermosensitivity (Larkindale and Huang, 2005; Larkindale et al., 2005). Exoge- nous application of these signaling agents to plants can also result in a degree of enhanced thermotolerance without an accompanying accumulation of Hsps (Larkindale and Knight, 2002). Thus, thermotolerance is affected by a complex network of changes in plants, only one of which is the production of Hsps (Larkindale and Vierling, 2008).

The SA-mediated pathway has been reported to protect potato, mustard, tobacco, tomato, bean, and Arabidopsis thaliana from heat stress (Dat et al., 1998; 2000; Larkindale and Knight, 2002; Lopez-Delgado et al., 1998; Senaratna et al., 2000). HS was found to induce SA-regulated pathogenesis-related 1 (PR1) transcripts, and the constitutive expression of PR1 protein mutant (cpr1-5) exhibited an enhanced thermotolerant phenotype (Clarke et al., 2004; 2009). This indicates that SA and PR1, originally defined as being involved in antipathogenic responses, can also promote heat tolerance in $A$. thaliana. The earlier study revealed that multiple bridging factor 1c (MBF1c) is a key regulator of thermotolerance in $A$. thaliana that functions upstream of SA and PR1 during heat stress. In addition, MBF1c-mediated thermotolerance is independent of Hsps expression (Suzuki et al., 2008).

Jasmonate $(\mathrm{JA})$ has been implicated in signaling in response to both biotic and abiotic stresses (Balbi and Devoto, 2008; Wasternack, 2006; 2007). Wounding and pathogen infection, or exposure to ozone, cause endogenous JA accumulation (Howe, 2004; Kanna et al., 2003; Rao et al., 2000; Vijayan, et al., 1998; Wasternack, 2006). Heat stress also induces the JA signal transduction pathway (Clarke et al., 2009). The relationship between JA- and SA- signaling has often been shown to be antagonistic. In $A$. thaliana, pathogen-induced SA accumulation is associated with the suppression of JA signaling (Spoel et al., 2003). In contrast, a recent finding has demonstrated that JA acts with SA to confer thermotolerance in $A$. thaliana, and that Hsps apparently play no role in JA-conferred thermotolerance in this species (Clarke et al., 2009).

The WRKY transcription factor superfamily has been suggested to have a key role in biotic and abiotic stress (Eulgem and Somssich, 2007; Miller et al., 2008). The A. thaliana WRKY superfamily consists of over 74 members, and is sub-divided into three groups on the basis of the number of WRKY (WRKYGQK) domains and the features of their zinc finger-like motif (Eulgem et al., 2000). Earlier reports have demonstrated

${ }^{1}$ College of Food Science and Nutritional Engineering, China Agricultural University, Beijing 100083, China, ${ }^{2}$ Xishuangbanna Tropical Botanical Garden, Chinese Academy of Sciences, Kunming 650223, China

*Correspondence: huanggwd@263.net (WH); ydq@xtbg.ac.cn (DY) 
that a number of $W R K Y$ genes are involved in the response to diverse sources of biotic stress (Dong et al., 2003; Eulgem and Somssich, 2007; Yu et al., 2001) and senescence (Gadjev et al., 2006; Jing et al., 2009). There is increasing evidence that several WRKY genes are involved in the response to various abiotic stresses, including heat (Miller et al., 2008). A WRKY transcription factor in tobacco (Nicotiana tabacum L.) has been found to respond to a combination of drought and HS (Rizhsky et al., 2002). Our previous studies have shown that $A$. thaliana WRKY25 is a positive regulator in thermotolerance ( $\mathrm{Li}$ et al., 2009). The expression of WRKY18, $-33,-40$, and -46 is elevated in MBF1C over-expressing plants, which possess enhanced thermotolerance compared with wild-type plants (Suzuki et al., 2005). Microarray analysis of $A$. thaliana hsf1a/hsf1b double knock-out mutants has revealed that nine of 60 analyzed WRKY genes are regulated by $\mathrm{HS}$, and among these nine, WRKY 7 is a HsfA1a/1b-dependent HS gene (Busch et al., 2005). Although several WRKY genes in $A$. thaliana are known to be influenced by HS, the roles of WRKY genes in thermotolerance during heat stress have been rarely reported.

WRKY39, a group II WRKY protein, is composed of 330 amino acids. Pathogen infection and SA can activate the expression of WRKY39 (Dong et al., 2003). Through microarray analysis, we found that WRKY39 was one of the most abundant $W R K Y$ transcripts in heat-treated wild-type plants. After 1 $\mathrm{h}$ heat stress at $42^{\circ} \mathrm{C}$, the transcript levels of WRKY39 increased by an average of 5.3-fold over non-treatment controls, and WRKY25 was up-regulated 4.9-fold (data not shown). Earlier findings that showed WRKY39 is a calmodulin (CaM)binding transcript factor with a protein structure close to WRKY7 (Park et al., 2005) allowed us to explore its thermotolerance function in $A$. thaliana. In the present study, we characterized the positive role of the $A$. thaliana WRKY39 transcription factor in plant responses to high temperature, by analyzing its potential upstream and downstream targets, and its loss-offunction and over-expression phenotypes. We suggest that WRKY39 regulates the cooperation between the SA- and JAactivated signaling pathways that mediate responses to heat stress.

\section{MATERIALS AND METHODS}

\section{Plant materials and growth conditions}

The $A$. thaliana mutants and wild-type plants used in this study are from the Columbia genetic background. The mutant lines coi1, ein2, and sid2 were from Prof. Zhixiang Chen (Purdue University, USA). T-DNA insertion mutants, including wrky39 (SALK_073483c), hot1 (SALK_036423c), and mbf1c (SALK_ $083813 \mathrm{C}$ ), were obtained from Arabidopsis Biological Resource Center (ABRC) (Alonso et al., 2003). Semiquantitative RT-PCR and Northern blot analyses were performed to further confirm the T-DNA insertion mutants. Procedures for growing plants were as previously described (Fu et al., 2009; Li et al., 2009).

\section{Generation of transgenic lines and plant transformation} The WRKY39 full cDNA linked to a vector pUNI was obtained from ABRC. To generate the 35S:WRKY39 construct, the cDNA was subcloned by ligating the Sacl-Sall fragment into the Sacl and Sall sites of pOCA30 (Chen and Chen, 2002) behind the CaMV $35 S$ promoter. For promoter- $\beta$-glucuronidase (GUS) fusion, the promoter region of WRKY39 gene was amplified by PCR from wild-type genomic DNA using the primers $5^{\prime}$-TTTGAGCTC-AACAATCGTGATTCGTGAT- $3^{\prime}$ and 5'-TTTCCATGGTTITC-TTCTACCCAAGTTCAGA-3' (underlined sections are Sacl and Ncol sites, respectively). The PCR product was restricted and inserted into pSJ131 upstream of the GUS gene. Then, a BamHI-Sacl fragment of pSJ131 containing the promoter of WRKY39, the GUS gene, and poly $(\mathrm{A})$ signal sequences was inserted into the BamHI and Sacl sites of pOCA28 (Du and Chen, 2000). The recombinant plasmids were introduced into Agrobacterium tumefaciens GV3101 and used to transform $A$. thaliana by the floral dip method (Clough and Bent, 1998). Transformed lines were selected for resistance to kanamycin $(50 \mu \mathrm{g} / \mathrm{ml})$. PCR and Northern blot analyses were performed to select the transgenic plants. Homozygous $T_{3}$ lines were obtained for phenotypic scoring under heat stress or by GUS staining.

\section{Histochemical GUS staining}

Plants were placed into staining solution containing $2 \mathrm{mM} 5-$ bromo-4-chloro-3-indolyl glucuronide (first dissolved in dimethyl formamide at $5 \mathrm{mg} \mathrm{ml}^{-1}$ ) in $50 \mathrm{mM}$ sodium-phosphate buffer, $\mathrm{pH}$ 7.0, $0.5 \mathrm{mM} \mathrm{K}_{4} \mathrm{Fe}(\mathrm{CN})_{6}, 0.5 \mathrm{mM} \mathrm{K}_{3} \mathrm{Fe}(\mathrm{CN})_{6}$, and $0.1 \%$ Triton X100. The samples were then placed under vacuum three times for 1 min each time for infiltration and incubated at $37^{\circ} \mathrm{C}$ overnight (Weigel and Glazebrook, 2002). Chlorophyll was removed by dipping in $75 \%$ ethanol.

\section{Heat stress treatments}

To examine the heat stress test response in seed germination, seeds were sown on water-saturated filter paper and treated at $45^{\circ} \mathrm{C}$ for $4 \mathrm{~h}$ immediately after removal from $4^{\circ} \mathrm{C}$ refrigeration. Thereafter they were allowed to germinate in a growth cabinet at $22^{\circ} \mathrm{C}$. Germination was recorded daily until no further germination was observed. To assess heat tolerance, seeds were geminated on $1 / 2$ MS medium. After 7 days, seedlings were transplanted into soil pots. Twenty-five-day-old plants grown in soil were exposed to heat stress at $48^{\circ} \mathrm{C}$ for $6 \mathrm{~h}$, returned to $22^{\circ} \mathrm{C}$ for 9 days, and then photographed. The 30-day assay was done by placing leaves from the same whorl of each 30-day-old plant into a 24-well plate, with $2.5 \mathrm{ml}$ of water in each well. The leaves were then heat-treated at $45^{\circ} \mathrm{C}$ for $4 \mathrm{~h}$ in a waterbath and left to recover at $22^{\circ} \mathrm{C}$ for 4 days. In all cases, the heat treatment was given in the dark, and the results for mutant and transgenic plants were compared with the wild-type plants on the same plate.

\section{Electrolyte leakage measurements}

Measurement of leaf electrolyte leakage (EL) was carried out essentially as described by Clarke et al. (2004). For experiments where EL was measured during heat treatment at $42^{\circ} \mathrm{C}$, the aerial parts of 3-week-old plants were placed into glass tubes containing $5 \mathrm{ml}$ of water and incubated in a waterbath at $42^{\circ} \mathrm{C}$ in the dark. The conductance of the water was measured at intervals during the heat treatment and calculated per milligram of fresh weight.

\section{Chemical treatment of plants}

SA was dissolved in water as a $100 \mathrm{mM}$ stock solution and adjusted to $\mathrm{pH} 6.5$ with $\mathrm{KOH}$. Methyljasmonate (MeJA) was dissolved in $50 \%$ ethanol as a $10 \mathrm{mM}$ stock solution and 1aminocyclopropane-1-carboxylic acid (ACC) was dissolved in water as a $10 \mathrm{mM}$ stock solution. Aerial parts of 3 -week-old $A$. thaliana soil-grown plants were incubated with $1 \mathrm{mM} \mathrm{SA}, 100$ $\mu \mathrm{M}$ MeJA, and $100 \mu \mathrm{M}$ ACC solution diluted from the stock.

\section{RNA extraction, cDNA synthesis, and semiquantitative RT-PCR analysis}

RNA was isolated from aerial parts of $A$. thaliana plants by standard protocols (Sambrook et al., 2001). The first-strand cDNA was synthesized from $2 \mu \mathrm{g}$ of DNase-treated RNA using 
A meeveaAnrsaieschgVlnllsQrtsdpesltvetgevVs KFKRVASLLTRGLGHGKFRSTNKFRSSFQHIFLESPICCGN DLSGDYTQVLAPEPLQMVPASAVYNEMEPKHQLGHPSLM LSHKMCVDKSFLELKPPPFRAPYQLIHNHQQIAYSRSNSGV NLKFDGSGSSCYTPSVSNGSRSFVSSLSMDASVTDYDRNSF HLTGLSRGSDQQHTRKMCSGSLKCGSRSKCHCSKKRKLR VKRSIKVPAISNKIADIPPDEYSWRKYGOKPIKGSPHPRGY YKC SSVRG QSAHT

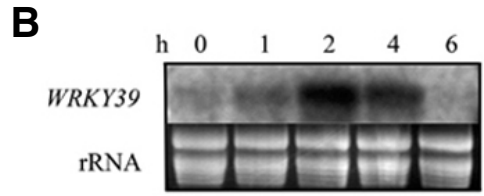

Fig. 1. Sequence of WRKY39 protein and accumulation of WRKY39 transcripts during heat stress in A. thaliana. (A) Amino acid sequence of WRKY39. One WRKY motif is indicated with the highly conserved WRKYGQK sequence and the residues forming the $\mathrm{C}_{2} \mathrm{H}_{2}$ zinc finger are underlined. The deduced amino acid sequence of the $\mathrm{C}$-region (conserved primary motif in WRKY group Ild members, CaM-binding domain) of $A$. thaliana WRKY39 is shown in white on a black background. (B) Northern blot of WRKY39 accumulation at $42^{\circ} \mathrm{C}$ for $0,1,2,4$, and $6 \mathrm{~h}$ in wild-type plants. The full length WRKY39 cDNA was used as a probe, and ribosomal $18 \mathrm{~S}$ RNA was used as the control for RNA loading.

M-MuLV reverse transcriptase (Fermentas, EU) with oligo(dT) 18 $_{18}$ primer, and $1 \mu \mathrm{l}$ of the cDNA was used in the subsequent PCR. The following gene-specific primers were used: WRKY39 (At3g04670) 5'-TGACTCTGTATCATCAAGTGTGA-3' and 5'AGTATTCTTGGAGGAAGTATGGA-3'; PR1 (At2g14610): 5'TCTTCCCTCGAAAGCTCAAG-3' and 5'-ACACCTCACTTTGGCACATC-3'; MBF1C (At3g24500): 5'-TCTCAATTCATCGACGATGC-3' and 5'-CGACATCGTTTAATCATTTCCC-3'; Hsp101 (At1g74310): 5'-GATTTGTTGAACGAAGTCGGTGT-3' and 5'ACCAGTTATGTAACGAGCAGACAGC-3'; APX1 (At1g07890): 5'-AGAGCTTAGCTAAGATGACGAAGAA-3' and 5'-TGATGGAAATCAGCAAAAGAGA-3'; Zat10 (At1g27730): 5'-TTAACTTAATGGCGCTCGAG-3' and 5'-ACAACTCTCAACAGTATACAAACGAA-3'; Hsp70 (At3g12580): 5'-AGGTTATCAAAGGAAGAGATCGA-3' and 5'-AGTAAACAAAAGCCAAAAGGCT-3'; Actin2 (At3g18780): 5'-TGTGCCAATCTACGAGGGTTT-3' and 5'-TTTCCCGCTCTGCTGTTGT-3'. The RevertAid ${ }^{\top \mathrm{M}}$ First Strand cDNA Synthesis Kit (Fermentas, EU) was used according to the manufacturer's protocol.

\section{Primer design and quantitative RT-PCR (qRT-PCR)}

Primers for qRT-PCR were designed and synthesized by TaKaRa. qRT-PCR was performed on the Roche LightCycler 480 machine using home-made $2 \times$ SYBR Green I master mix as per manufacturer's instructions. Each $20 \mu \mathrm{l}$ reaction contained $1 \mu \mathrm{l}$ of cDNA and $10 \mu \mathrm{M}$ of each gene-specific primer. Actin2 (At3g18780) was used as the reference gene internal control for comparison of the target gene transcripts amplified per cDNA sample. The primer sequences of Actin2 were as described above. The following primers were used for target gene amplification: WRKY39 (At3g04670): 5'-TGCGGAAGTCGAAGCAAATGTCA-3' and 5'-CGTGGATGCGGTGAACCCTTTAT3'; PR1 (At2g14610): 5'-AACTACAACTACGCTGCGAACA-3' and 5'-GTTACACCTCACTTTGGCACAT-3'; MBF1c (At3g24500): 5'-ATGCCGAGCAGATACCCAGGAGC-3' and 5'-TTAACCGTTTGAACCGCGACACC-3'. The LightCycler Software 480 (Roche Diagnostics) relative quantification analysis module was used to determine the relative fold expression changes between samples. All PCRs were performed under the following conditions: $4 \mathrm{~min}$ at $95^{\circ} \mathrm{C}$ followed by 50 cycles of $30 \mathrm{~s}$ at $95^{\circ} \mathrm{C}, 30 \mathrm{~s}$ at $58^{\circ} \mathrm{C}$, and $20 \mathrm{~s}$ at $72^{\circ} \mathrm{C}$, in LightCycler capillaries (Roche Diagnostics). The specificity of amplicons was verified by melting curve analysis $\left(60-95^{\circ} \mathrm{C}\right)$ after 50 cycles. At least three biological replicates for each sample were used for qRT-PCR analysis and at least two technical replicates were analyzed for each biological replicate.

\section{Northern blot analysis}

$\left[\alpha-{ }^{32} \mathrm{P}\right] \mathrm{dATP}(>3000 \mathrm{Ci} / \mathrm{mmol})$ was obtained from Beijing FuRui Biological Technology Company. Total RNA (10 or $20 \mu \mathrm{g}$ ) was separated on agarose-formaldehyde gels and transferred onto nylon membranes, which were hybridized and washed following standard procedures (Sambrook et al., 2001). Transcripts for WRKY39 were detected using full-length WRKY39 cDNA as a probe, which was labeled by $\left[\alpha^{-32} P\right]$ dATP using the TaKaRa Random Primers DNA Labeling System.

\section{RESULTS}

\section{WRKY39 is induced by heat stress}

A. thaliana WRKY39 (At3g04670) encodes a protein of 330 amino acids with a molecular weight of $36.718 \mathrm{kD}$ and an isoelectric point of 9.78 (Fig. 1A). Based on the presence of one WRKY domain (Fig. 1A) and additional short conserved structural motifs (Eulgem et al., 2000), WRKY39 is classified as a group Ild WRKY protein. An earlier study has shown that WRKY39 is a calmodulin-binding protein (Fig. 1A; Park et al., 2005).

According to microarray experiments, the WRKY39 gene is expressed at low levels in healthy, non-stressed wild-type plants. However, after $1 \mathrm{~h}$ heat stress at $42^{\circ} \mathrm{C}$, the transcript levels of WRKY39 increased by an average of 5.3-fold over non-treatment controls. To confirm the microarray results, we examined the transcript levels of WRKY39 in heat-treated plants using RNA blots. The transcript levels of WRKY39 increased rapidly and strongly within 1-2 $\mathrm{h}$ after heat treatment, and then returned to basal levels within $6 \mathrm{~h}$ (Fig. 1B). This expression pattern shows that HS might play a role in significantly and transiently up-regulating transcription factor activity toward the WRKY39 promoter, indicating that WRKY39 is involved in responses to heat stress.

\section{Promoter-GUS reporter fusion}

To test the ability of the putative promoter region from WRKY39 to direct heat stress-responsive WRKY39 expression, we fused a genomic fragment from $1.5 \mathrm{~kb}$ upstream of the translational start codon to a GUS reporter gene.

Five independent $T_{3}$ transgenic $A$. thaliana lines carrying the WRKY39 promoter-GUS fusion were examined, and representative consensus expression patterns are described in Fig. 2. In non-stressed plants harboring the $P_{\text {wrky } 39}$ :GUS fusion, we detected moderate expression in the roots and veins of cotyledons of 3-d seedlings (Fig. 2A), and in the veins of fresh leaves and roots in both 7-day seedlings and 21-day plants (Figs. 2B and $2 \mathrm{C}$ ). Within the inflorescences, moderate to strong GUS expression was observed in stamens, petals, stems, and tips and nodes of siliques under normal growth conditions (Fig. 2D).

Having established the basal pattern of GUS expression for the $P_{\text {wrky } 39}$ :GUS fusion construct, we next examined GUS expression in 21-day plants heat-treated at $42^{\circ} \mathrm{C}$ for $4 \mathrm{~h}$, or in the presence of SA, MeJA, or ACC, the immediate precursor of ethylene), compared with non-stressed or mock-treated controls 


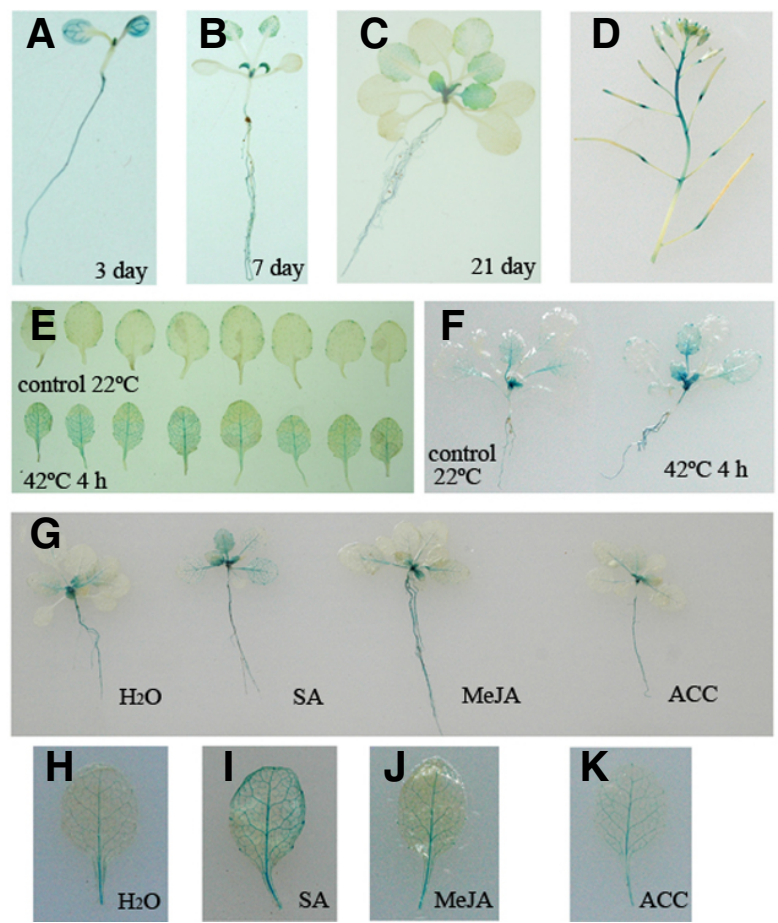

Fig. 2. Histochemical localization of GUS activity in representative $\mathrm{T}_{3}$ transgenic $A$. thaliana plants harboring WRKY39 promoter-GUS constructs. (A-D) WRKY39-GUS expression in non-stressed, representative transgenic plants. (A) 3-day-old seedlings; (B) 7-day-old seedlings; (C) 21-day-old plants; (D) inflorescence and siliques. (EF) WRKY39-GUS expression during heat stress. (E) 21-day-old WRKY39-GUS plants were incubated at $42^{\circ} \mathrm{C}$ for 0 or $4 \mathrm{~h}$, and leaves were stained; $(F)$ 21-day-old WRKY39-GUS plants were incubated at $42^{\circ} \mathrm{C}$ for 0 or $4 \mathrm{~h}$, and whole plants were stained. (G) GUS staining patterns in 21-day-old WRKY39-GUS plants treated with $\mathrm{H}_{2} \mathrm{O}, 1 \mathrm{mM} \mathrm{SA}, 0.1 \mathrm{mM}$ MeJA, or $0.1 \mathrm{mM} \mathrm{ACC}$ for $4 \mathrm{~h}$. $(\mathrm{H}-\mathrm{K})$ GUS staining pattern in 21-day-old leaves of WRKY39-GUS plants with different treatments for $4 \mathrm{~h}$ : (H) $\mathrm{H}_{2} \mathrm{O}$; (I) $1 \mathrm{mM} \mathrm{SA}$; (J) $0.1 \mathrm{mM}$ MeJA; (K) $0.1 \mathrm{mM} \mathrm{ACC}$.

(Figs. 2E-2K). Although a basal level of GUS expression was detected throughout the plant, an increase in GUS expression was detected following $4 \mathrm{~h}$ of heat stress (Figs. $2 \mathrm{E}$ and $2 \mathrm{~F}$ ). We also observed an apparent increase in GUS staining intensity in leaf veins of $P_{\text {wrkyzg: }}$ GUS lines treated with SA, and a slight increase in lines treated with MeJA. Water and ACC treatment did not induce GUS expression (Figs. 2G-2K).

\section{Identification of wrky39 knock-down mutants and generation of WRKY39-overexpressed transgenic A. thaliana plants}

To analyze the role of WRKY39 in thermotolerance, we identified one T-DNA knock-down mutant for WRKY39. Homozygous wrky39 (SALK_073483c) mutants contain a T-DNA insertion at the beginning of the $5^{\prime}$ untranslated region, 596 nucleotides upstream of the start codon (Fig. 3A). We performed RTPCR to compare wild-type plants and wrky39 mutants for induced accumulation of WRKY39 transcripts during heat stress at $42^{\circ} \mathrm{C}$. WRKY39 transcripts of the expected induction level were observed in wild-type plants after heat treatment. In contrast, the basal level of WRKY39 transcripts was apparently reduced, and heat stress did not induce WRKY39 expression in wrky39 mutants (Fig. 3B). When grown under normal condi-
A

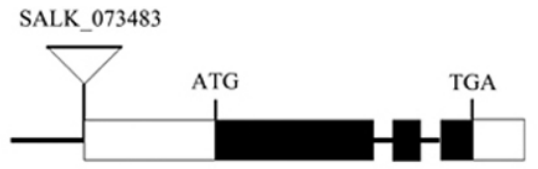

B
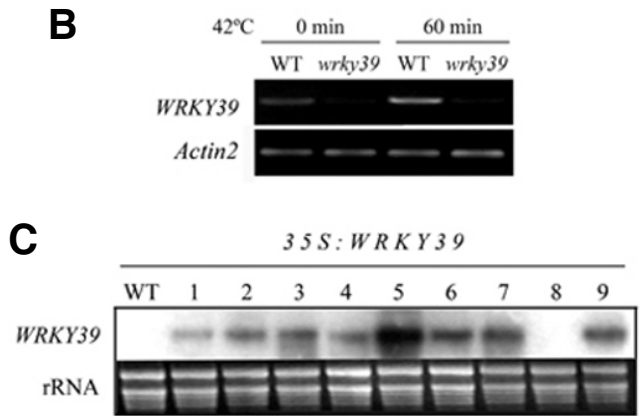

Fig. 3. Characterization of wrky39 T-DNA knock-down mutants and WRKY39 over-expressing plants. (A) Diagram of the WRKY39 gene and its T-DNA insertion mutant. (B) RT-PCR analysis of the wrky39 mutant line. Leaves from wrky39 mutant and wild-type plant were harvested before heat stress or after treatment at $42^{\circ} \mathrm{C}$ for 60 min. Total RNA was isolated and Actin2 amplification was used as constitutive control. (C) Northern blot analysis of WRKY39 in wildtype and transgenic plants. Each lane was loaded with $10 \mu \mathrm{g}$ of RNA prepared from 3-week-old plants. Ribosomal 18S RNA was used as the control for RNA loading.

tions, both seedling and adult wrky39 mutants showed no morphological or developmental difference compared with wild-type plants (data not shown).

To further investigate the function of WRKY39, we constructed and analyzed transgenic $A$. thaliana plants that constitutively over-express WRKY39. The full-length WRKY39 cDNA was cloned behind the CaMV $35 S$ promoter and used to transform $A$. thaliana plants. Northern blot analysis identified several transgenic plants that contained elevated levels of WRKY39 transcript in the absence of heat stress (Fig. 3C). Two transgenic lines (lines 5 and 6 in Fig. 3C) were selected for further study. Line 5 showed a markedly higher level of expression of WRKY39 compared with the wild-type, and line 6 also showed a higher level of expression than the wild-type (Fig. 3C). Analysis of $T_{3}$ homozygous plants from both lines revealed no differences in growth or development from that of wild-type plants (data not shown).

\section{The wrky39 mutants and over-expression of WRKY39 result in altered responses to heat stress}

We first examined the germination of wrky39 and transgenic 35S:WRKY39 seeds. Seeds were exposed to a temperature of $45^{\circ} \mathrm{C}$ for $4 \mathrm{~h}$ after vernalization for three days at $4^{\circ} \mathrm{C}$ and then returned to $22^{\circ} \mathrm{C}$ for recovery. Radicle emergence was scored daily until no further germination of 35S:WRKY39 was observed. At 3 days after heat treatment, 35S:WRKY39 lines already showed 2.5-4-fold increase in germination than wildtype seeds, whereas germination of wrky39 seeds was nearly one-half that of the wild-type (Fig. 4). By 5 days after heat treatment, 35S: WRKY39 seeds exhibited $84-95 \%$ germination, while wild-type and wrky39 showed approximately $63 \%$ and $47 \%$ germination, respectively (Fig. 4). Although some of the wrky39 seeds were finally able to germinate, wild-type seeds displayed higher germination over time (Fig. 4; wild-type and wrky39, $P<0.05)$. 35S:WRKY39 seeds showed tolerance to 
A

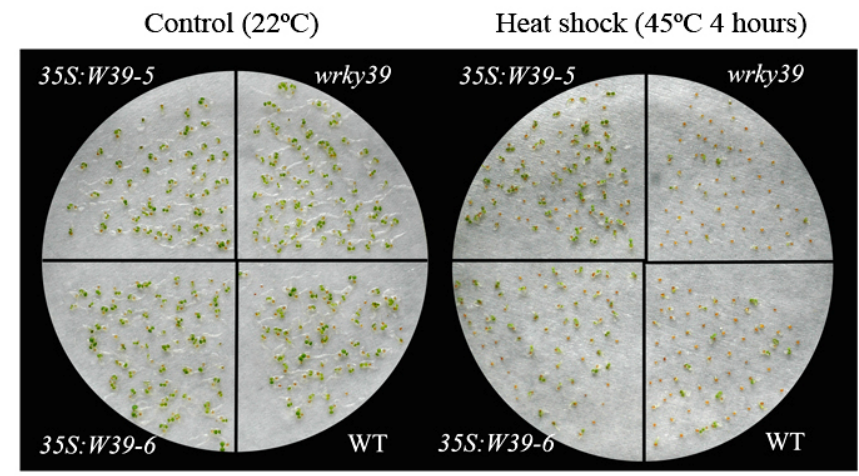

B

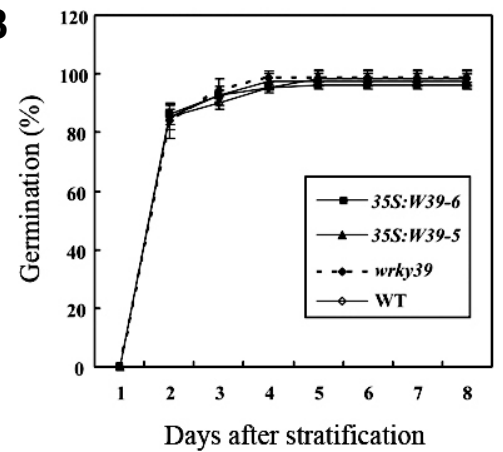

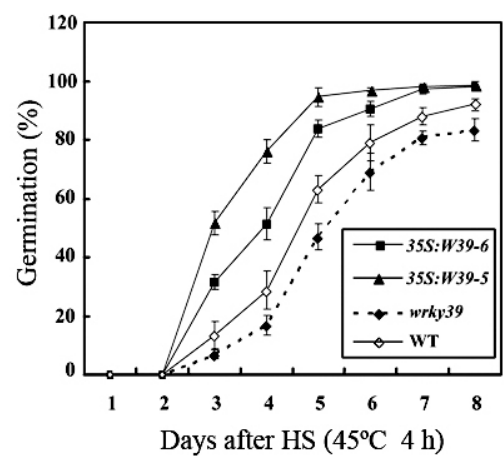

Fig. 4. Response of the wrky39 mutants and 35S:WRKY39 lines to heat stress during seed germination. Wild-type, wrky39, 35S:WRKY39-5, and 35S:WRKY39-6 seeds were sown on watersaturated filter paper. Seeds were treated at $45^{\circ} \mathrm{C}$ for $4 \mathrm{~h}$ or incubated at $22^{\circ} \mathrm{C}$ (control) immediately after removal from $4^{\circ} \mathrm{C}$ ( 3 days in the dark), and then maintained at $22^{\circ} \mathrm{C}$. Germination was assessed at the indicated intervals. (A) Illustration of representative seeds/seedlings 4 days after heat treatment. (B) The percentage of radicle emergence was recorded daily until no further germination of 35S:WRKY39 was observed. The data are shown as mean $\pm \operatorname{SD}(n=4)$. Each replicate consisted of 50-70 seeds. The Mann-Whitney U-test was used for data analysis.
HS (Fig. 4; wild-type and 35S:W39-5, $P<0.01$; wild-type and 35S:W39-6, $P<0.05)$. Moreover, 35S:W39-5 caused greater seed germination than 35S:W39-6 (Fig. 4; 35S:W39-5 and 35S:W39-6, $P<0.05)$. Heat treatment did not significantly alter seedling viability after ger-mination (data not shown), but did impair development (Fig. 4).

We also used 25-day-old plants to compare the degree of thermotolerance among wrky39 mutants, wild-type plants, and 35S:WRKY39 lines. Plants were directly subjected to heat stress $\left(48^{\circ} \mathrm{C}\right)$ for $6 \mathrm{~h}$, and then returned to $22^{\circ} \mathrm{C}$ for recovery. Nine days later, surviving plants could be clearly identified by the presence of newly emerging leaves, while non-surviving plants exhibited complete etiolation, loss of turgor, and arrested leaf formation (Fig. 5A). The degree of thermotolerance correlated with the level of WRKY39 expression, in which the 35S:W39-5 and 35S:W39-6 plants showed higher levels of expression and greater thermotolerance than wrky39 mutant and wild-type plants (Fig. 5A).

Thermosensitivity of wrky39 mutants and enhanced thermotolerance of 35S:WRKY39 lines were also observed in a 30-d assay (Fig. 5B). Leaves of the same whorl were removed from 30- $\mathrm{d}$ plants and heated to $45^{\circ} \mathrm{C}$ for $4 \mathrm{~h}$ in a waterbath, and then left to recover at $22^{\circ} \mathrm{C}$ for 4 days. Figure $5 \mathrm{~B}$ illustrates that reduction of leaf chlorophyll content by heat stress was aggravated significantly in the wrky39 mutants in comparison to the wild-type and 35S:WRKY39 plants.

We extended our assay to measure EL in the wrky39 mutants, wild-type plants, and 35S:WRKY39 lines. EL represents a rapid, sensitive and quantitative method to assess the effects of stress on plant cells (Clarke et al., 2004; 2009; Hong et al., 2003; Howarth et al., 1997). Significantly less EL was detected for 35S:WRKY39 compared with wild-type during heat stress after $3 \mathrm{~h}$ (Fig. 5C; wild-type and 35S:W39-5, $P<0.01$; wild-type and 35S:W39-6, $P<0.05)$. In addition, heat treatment of wrky39 at $42^{\circ} \mathrm{C}$ for $4 \mathrm{~h}$ resulted in nearly 1.3 -fold greater EL than in the wild-type (Fig. 5C)

On the basis of the assays described above, constitutive over-expression of WRKY39 in A. thaliana enhances thermotolerance, while reduced expression of WRKY39 leads to thermosensitivity, indicating that this gene has a positive role in thermotolerance.

\section{Expression of heat stress defense genes in wrky39 mutants and WRKY39 over-expressing plants}

The expression of a number of heat stress-related genes was analyzed in wrky39 mutants, wild-type plants, and WRKY39 over-expressing lines (lines 5 and 6 ) to further confirm the role of WRKY39 during heat stress. The expression patterns of wellcharacterized heat stress-responsive marker genes were analyzed by semi-quantitative RT-PCR or qRT-PCR. Total RNA of wrky39, wild-type, and 35S:WRKY39 were isolated after heating at $42^{\circ} \mathrm{C}$ for $0,30,60$, or $120 \mathrm{~min}$.

A. thaliana Hsp70 and Hsp101 are well-known HS-induced genes that play important roles in thermotolerance (Larkindale and Huang, 2005; Larkindale et al., 2005). A. thaliana APX1 is a key $\mathrm{H}_{2} \mathrm{O}_{2}$ removal enzyme (Panchuk et al., 2002; Pnueli et al., 2003), and the transcriptional regulator Zat10 is a key regulator of reactive oxygen species signaling (Miller et al., 2008). In our study we found that Hsp70, Hsp101, and APX1 expression patterns were similar in wrky39 mutants, wild-type plants, and 35S:WRKY39 lines after HS treatment at $42^{\circ} \mathrm{C}$ (Fig. 6A). Although the 35S:W39-5 line had higher levels of Zat10 transcript than wild-type plants after heat treatment, the expression of Zat10 was not significantly different in wrky39 mutants, wildtype plants, or the 35S:W39-6 line (Fig. 6A).

Recent studies have shown that SA-mediated pathway promotes thermotolerance in plants (Clarke et al., 2004; 2009; Dat et al., 1998; 2000; Larkindale and Knight, 2002; Lopez-Delgado et al., 1998; Senaratna et al., 2000). To determine whether the altered responses of the wrky39 mutants and the transgenic 
A

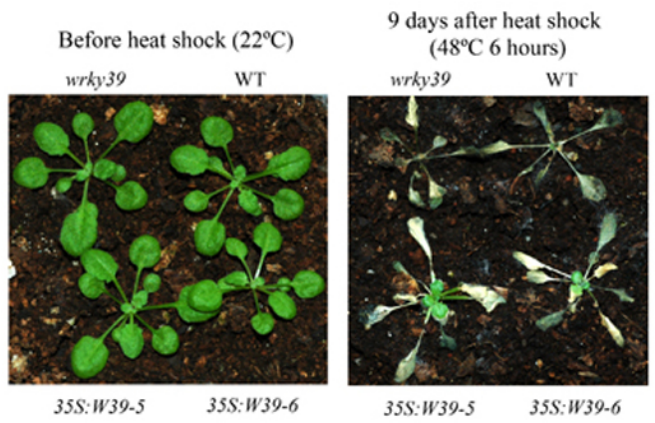

B

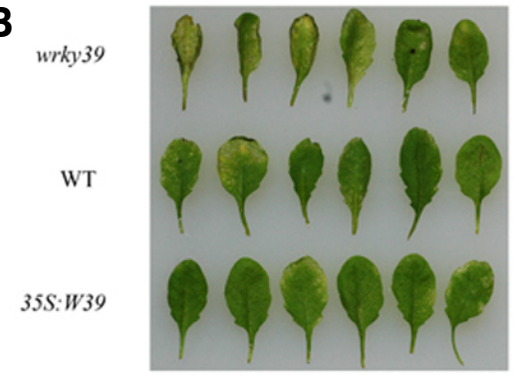

C

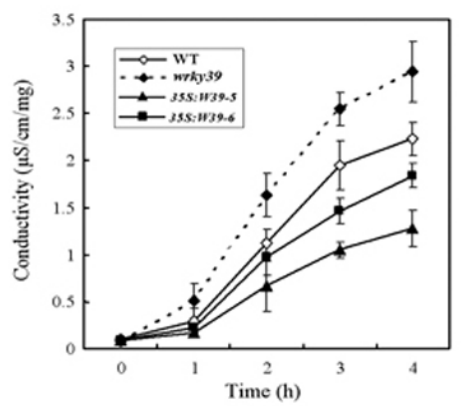

Fig. 5. Altered responses of wrky39 mutants and 35S:WRKY39 plants to heat stress. (A) 25-day-old plants of the wild-type, wrky39 mutant, 35S:WRKY39-5, and 35S:WRKY39-6 were treated at $48^{\circ} \mathrm{C}$ for $6 \mathrm{~h}$. Photographs were taken before heat treatment or after a 9 days recovery period at $22^{\circ} \mathrm{C}$. The experiments were repeated four times with similar results and a representative result is shown. (B) Photograph of leaves removed from 30-day-old wrky39 mutant, wild-type, 35S:WRKY39-5, and 35S:WRKY39-6 plants, heated to $45^{\circ} \mathrm{C}$ for $4 \mathrm{~h}$ in a waterbath and left to recover for 4 days. The experiments were repeated four times with similar results and a representative result is shown. (C) The assay of EL measurement was done with 21-day-old seedlings grown in soil. The aerial parts of wrky39 mutant, wild-type, 35S:WRKY39-5, and 35S:WRKY39-6 plants were incubated at $42^{\circ} \mathrm{C}$ in a waterbath, transferred to $22^{\circ} \mathrm{C}$ at the indicated time-point and the conductivity of the bathing solution was monitored. The data are shown as means from three experiments, each with five plants per treatment. Bars are presented as $\mathrm{SD}(n=15)$. The Mann-Whitney $U$-test was used for data analysis.

35S:WRKY39 plants to heat stress are related to altered SAmediated mechanisms, we examined the expression of two SA-related genes after heat treatment. $A$. thaliana MBF1c and $P R 1$ are heat-inducible genes associated with the SA pathway in thermotolerance; $M B F 1 c$ is also required for thermotolerance independent of Hsfs and Hsps, and functions upstream of SA and $P R 1$ (Suzuki et al., 2008). Figure 6 shows that the expression of $P R 1$ and $M B F 1 c$ was clearly induced in wild-type plants subjected to heat stress, in agreement with previous reports (Clarke et al., 2004; 2009; Suzuki et al., 2008). The expression of PR1 and MBF1C was also induced by heat stress in wrky39 mutants, with similar kinetics to that observed in wild-type plants. However, the fold-induction of PR1 and MBF1c expression in wrky39 mutants was apparently lower than in wild-type plants at each time-point after being transferred to $42^{\circ} \mathrm{C}$, particularly at $60 \mathrm{~min}$. At this point, the expression of $P R 1$ and MBF1c in wrky39 mutants was nearly 5- and 1.25-fold reduced, respectively, compared with wild-type plants (Fig. 6). Consistent with the thermotolerant phenotype, we consistently observed an increase in the inducible expression of $P R 1$ and $M B F 1 C$ in transgenic 35S:WRKY39 plants relative to wild-type plants (Fig. 6). In non-stressed plants, wrky39 mutants showed 2.8-fold lower $P R 1$ expression than wild-type plants, and the expression of $P R 1$ in 35S:WRKY39 lines was moderately increased compared with wild-type plants (Figs. 6A and 6B). No differences in MBF1c expression were observed among unstressed mutant, wild-type, and over-expressing plants, indicating that WRKY39 alone is not sufficient for altered MBF1c expression (Figs. 6A and $6 \mathrm{C}$ ).

Taken together, these results indicate that WRKY39 is a positive regulator of these SA-related heat stress defense genes. The expression of Hsp70, Hsp101, APX1, and Zat10 was the same in wrky39 mutants, wild-type plants, and 35S:WRKY39 lines, suggesting that WRKY39-mediated thermotolerance is independent of Hsps and heat-induced oxidative stress pathways.

\section{Expression of WRKY39}

To further investigate whether WRKY39 expression is influenced by altered expressions of Hsp101 and MBF1c during heat stress, WRKY39 expression was checked in hot1 (SALK_036423c) and mbf1c (SALK_083813c) mutants. Figures $7 \mathrm{~A}$ and $7 \mathrm{D}$ show that in wild-type plants, hot1, and mbf1c mutants, the expression of WRKY39 was induced by heat stress, reaching peak expression after $60 \mathrm{~min}$. At the same time, basal WRKY39 expression was slightly reduced and induced WRKY39 expression was modestly increased in hot1 and mbf1c mutants compared with wild-type plants (Figs. 7A and 7D). It appears that moderately increased expression of WRKY39 in hot1 and mbf1c mutants compensates for the absence of Hsp101 and MBF1c during heat stress.

We also analyzed WRKY39 induction in wild-type plants treated with SA, ACC, or MeJA. The WRKY39 gene was induced rapidly and strongly by SA (Figs. 7B and 7E), consistent with a previous report by Dong et al. (2003). The transcript levels of WRKY39 increased rapidly but modestly within 1-8 $\mathrm{h}$ after treatment with MeJA, and returned to basal levels within $24 \mathrm{~h}$ (Figs. 7B and 7E). ACC treatment did not induce WRKY39 expression (Figs. 7B and 7E). SA was by far the most potent inducer of WRKY39 transcript accumulation among the defense signal molecules we tested (Figs. 7B and 7E). These results agree with the GUS expression patterns (Figs. 2G-2K).

To determine which signaling pathways are involved in the heat-induced expression of WRKY39, its expression was studied in a set of mutants that are defective in various defense response pathways including JA signaling (coi1), ET response (ein2), and SA biosynthesis (sid2). All of these mutants exhibit thermosensitivity (Larkindale et al., 2005). During heat stress, WRKY39 expression was reduced in all of these mutants compared with the wild-type (Figs. 7C and 7F). WRKY39 expression was lower in coi1 and sid2 mutants than in ein2 mutants, indicating that COI1 and SID2, which are required for JA signaling and SA biosynthesis, respectively, induce expression of the WRKY39 gene during heat stress. 
A

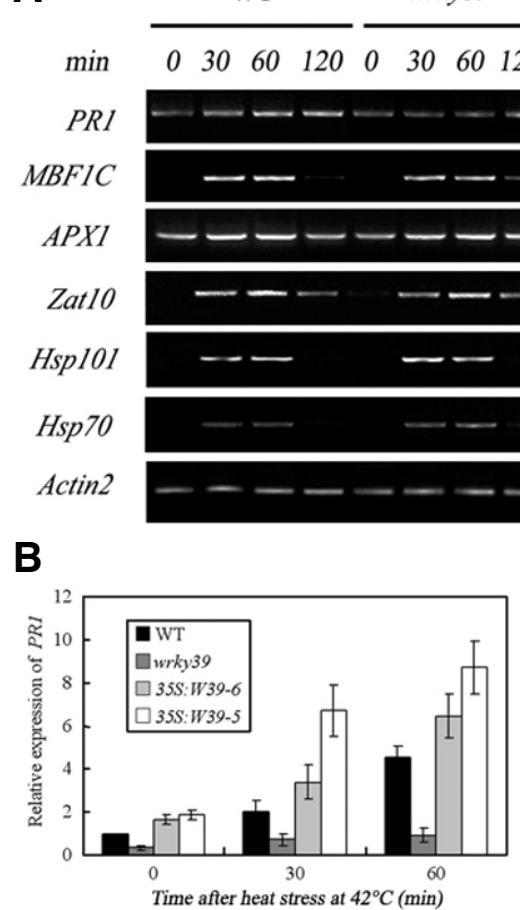

$\begin{array}{llllllllllllllllllllllllll}0 & 30 & 60 & 120 & 0 & 30 & 60 & 120 & 0 & 30 & 60 & 120 & 0 & 30 & 60 & 120\end{array}$

C

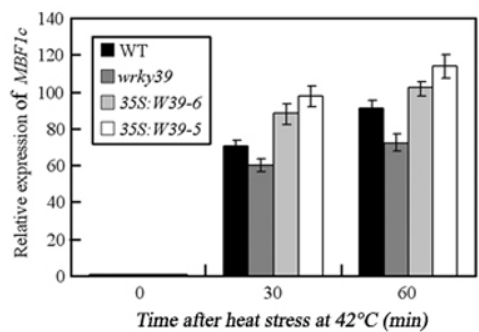

Fig. 6. Expression of heat-inducible and oxidative stress-responsive genes in wrky39 mutants and 35S:WRKY39 plants. (A) Semi-quantitative RT-PCR analysis of wrky39 mutant, wild-type, and 35S: WRKY39 plants. Total RNA was isolated from 21day-old plants exposed to $42^{\circ} \mathrm{C}$ for $0,30,60$, and 120 min. Actin2 amplification was used as a constitutive control. RT-PCR was repeated three times using RNA extracted from three independent experiments and similar results were obtained. (B) qRT-PCR analysis of $P R 1$ expression. (C) qRT-PCR analysis of MBF1C expression. In (B) and (C), the expression of target genes (PR1 and MBF1C) was detected in 21-day-old wrky39 mutant, wild-type, and 35S:WRKY39 plants treated at $42^{\circ} \mathrm{C}$ for 0,30 , or 60 min. Actin2 was used as an internal control and gene expression was normalized to the wild-type unstressed expression level, which was assigned a value of 1 . Data represent the average of three independent experiments \pm SD.

\section{DISCUSSION}

Here, we analyzed the roles of the A. thaliana WRKY39 gene in response to heat stress, extending observations made in our previous study (Li et al., 2009). Evidence that the heat stressinduced WRKY39 gene (Fig. 1) functions as a positive regulator in thermotolerance comes from analysis of transgenic overexpression lines and a T-DNA insertion mutant. The wrky39 knock-down mutants exhibited enhanced thermosensitivity by reduced germination, decreased survival, and enhanced $E L$ in heat-treated seeds or plants (Figs. 4 and 5). Transgenic plants constitutively expressing WRKY39, on the other hand, were more resistant to high temperature stress than wild-type plants (Figs. 4 and 5). Thus, mutations and constitutive WRKY39 over-expressing lines had contrasting effects in response to heat stress. In addition, we observed that the enhanced thermosensitivity of wrky39 mutants was associated with reduced expression of $P R 1$ and MBF1c (Fig. 6), two heat-related genes often associated with SA-mediated pathways (Suzuki et al., 2008). PR1 and MBF1c were expressed at relatively higher levels in WRKY39 over-expressing plants than in wild-type plants after heat treatment (Fig. 6). A. thaliana mutants sid2 and npr1, defective in SA biosynthesis or signaling, display reduced heat tolerance (Clarke et al., 2004; 2009), indicating that SAmediated signaling plays a vital role in thermotolerance in plants. Our data suggest that WRKY39 might play a positive role in SAmediated signaling pathways, and that over-expression of the gene could have a positive impact on SA-mediated heat stress defense mechanisms, increasing plant resistance to high temperatures. Moreover, because the expression of $P R 1$ was reduced more than that of MBF1c in wrky39 mutants (Fig. 6) and a recently reported model (Suzuki et al., 2008), we speculate that WRKY39 is likely to act upstream of $P R 1$ and downstream of MBF1C.

It should be noted that the basal expression of WRKY39 was apparently increased in 35S:WRKY39 lines, but was very low in wild-type (Fig. 3C). However, expression of the putative target gene (MBF1C) was not significantly induced in WRKY39 overexpression lines, although it was slightly increased compared with the wild-type (Fig. 6). This indicates that WRKY39 alone may not be sufficient to alter the expression of MBF1c. Meanwhile, as a member of a large gene family, WRKY39 may have functional overlap with other WRKY genes, as explained by the failure to observe stronger phenotypes in wrky39 mutants during heat stress (Figs. 4 and 5). Indeed, phylogenetic analysis based on WRKY protein structures has revealed that WRKY39 and several other WRKY proteins, including WRKY74 and WRKY21, share very similar protein structures (Dong et al., 2003). Therefore, the expression of WRKY74 and WRKY21 might compensate for the absent function of WRKY39. Isolation of the loss-of-function mutants for these similar WRKY proteins and construction of composite mutants for multiple WRKY genes should reveal whether these structurally related WRKY proteins perform overlapping functions in thermotolerance (Zheng et al., 2006).

Using the $P_{\text {wrky } 39}$ :GUS fusion, we detected inducibility of GUS expression by heat $\left(42^{\circ} \mathrm{C}\right), \mathrm{SA}$, and MeJA. The GUS expression patterns were generally consistent with the mRNA accumulation patterns (Figs. 2, 7B, and 7E). This suggests that regulatory elements exist within the promoter regions examined in our study, and that the sensitivity of the reporter system is sufficient to detect moderate changes in various treatments.

The proposed role of WRKY39 as a positive regulator of SAdependent heat stress defense pathways can also be deduced from the rapid and strong induction of WRKY39 expression by SA (Figs. 7B, 7E, and 2G; Dong et al., 2003). Comparisons between the three established defense signal molecules showed that only SA induced WRKY39 at high levels, and that JA or ET did not (Figs. 7B, 7E, and 2G). Moreover, SA was required for heat-induced WRKY39 expression, as shown by the significantly reduced induction of WRKY39 in sid2 mutant plants (Figs. 7C and 7F). As WRKY39 expression was also 
A

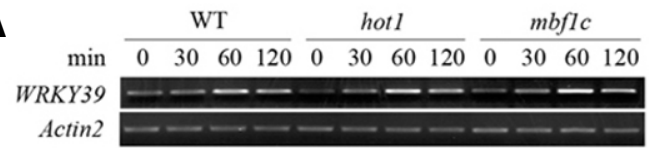

B

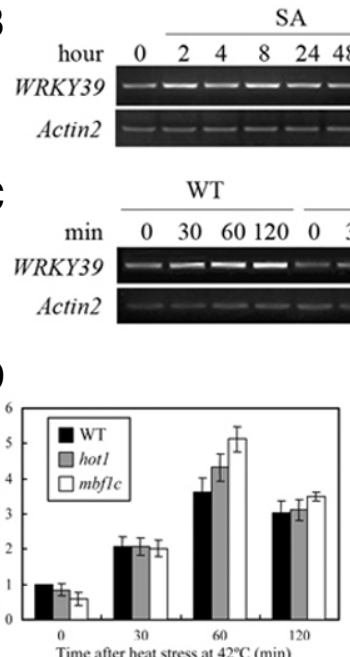

$\mathrm{A}$

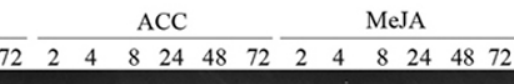

- - - - - - - - -

coil ein2 sid2

$\begin{array}{llllllllll}60 & 120 & 0 & 30 & 60 & 120 & 0 & 30 & 60 & 120\end{array}$

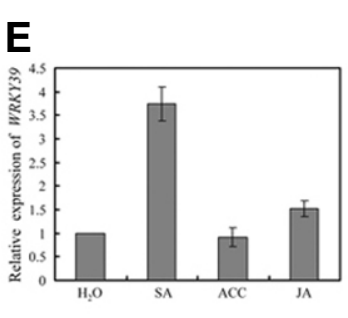

$\mathbf{F}$

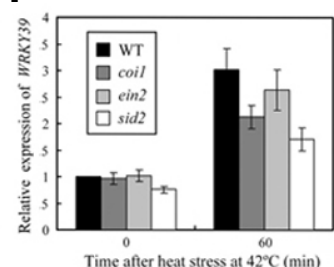

Fig. 7. Expression of WRKY39. (A) RT-PCR analysis of WRKY39 expression in wild-type, hot 1 , and mbf1c mutants during heat treatment at $42^{\circ} \mathrm{C}$ for $0,30,60$, and $120 \mathrm{~min}$. (B) Induced WRKY39 expression in wild-type plants incubated with $1 \mathrm{mM} \mathrm{SA}, 0.1 \mathrm{mM}$ MeJA, or $0.1 \mathrm{mM} \mathrm{ACC}$. Leaves were harvested at the indicated times after treatment and used for preparation of total RNA and RT-PCR. (C) Expression of WRKY39 in wildtype, coi1, ein2, and sid2 plants during heat treatment at $42^{\circ} \mathrm{C}$ for $0,30,60$, and $120 \mathrm{~min}$. In (A), (B), and (C), Actin2 amplification was used as constitutive control. The experiments were repeated three times using RNA extracted from three independent experiments and similar results were obtained. (D-F) qRTPCR analysis of WRKY39 expression. (D) Expression of WRKY39 in wild-type, hot1, and mbf1c mutants during heat treatment at $42^{\circ} \mathrm{C}$ for $0,30,60$ and $120 \mathrm{~min}$; (E) WRKY39 expression in wild-type plants incubated with $\mathrm{H}_{2} \mathrm{O}, 1 \mathrm{mM}$ SA, $0.1 \mathrm{mM}$ MeJA, or $0.1 \mathrm{mM}$ ACC for $8 \mathrm{~h}$; (F) Expression of WRKY39 in wild-type, coi1, ein2, and sid2 plants exposed heat stress at $42^{\circ} \mathrm{C}$ for 0 or $60 \mathrm{~min}$. In (D), (E), and (F), Actin2 was used as an internal controt and gene expression was normalized to the wild-type unstressed expression level, which was assigned a value of 1 . Data represent the average of three independent experiments \pm SD. The $A$. thaliana mutants and wild-type plants used in the experiments above were 3 weeks old. slightly induced by JA, and moderately decreased in coi1 during heat stress, JA is a possible candidate for one of the signal molecules that mediate heat- and SA- induced expression of WRKY39. Because JA acts with SA to confer thermotolerance in $A$. thaliana (Clarke et al., 2009), the induction of WRKY39 through JA-mediated signaling mechanisms is consistent with its demonstrated role in thermotolerance. Although WRKY39 expression was unaffected by ACC treatment, heat-induced expression of this gene was slightly reduced in ein2 mutants. Therefore, the role of ET in WRKY39 expression remains unclear.

The role of WRKY39 as a positive regulator in thermotolerance is also consistent with possible regulation of WRKY39 by calcium, a second messenger in $A$. thaliana. Calmodulin (CaM) interacts with the WRKY39 protein (Park et al., 2005) and calcium is involved in HS in plants through CaM (Liu et al., 2008; Zhang et al., 2009). Calcium does not appear to be involved in the classic HS signaling pathways, i.e. the induction of Hsps (Gong et al., 1997), which is similar to WRKY39 in heat stress (Fig. 6). Thus, WRKY39 may function as a downstream component of the CaM-mediated calcium signaling pathway during heat stress. Given that the WRKY39 protein may act as a regulatory node in thermotolerance by linking the SA, JA, and calcium signaling pathways, establishing the mechanism(s) for WRKY39-conferred thermotolerance is an important future task.

\section{ACKNOWLEDGMENTS}

We thank Dr. Zhixiang Chen (Department of Botany and Plant Physiology, Purdue University) for Arabidopsis coi1, ein2, and sid2. We are grateful to Dr. Douglas Schaefer for his critical reading of the manuscript. This work was supported by the Science Foundation of Ministry of Agriculture of the People's Republic of China (2009ZX08009-066B) and the National Natural Science Foundation of China (Nos. 30611468, 30871747, 30771223, and 90817003).

\section{REFERENCES}

Alonso, J.M., Stepanova, A.N., Leisse, T.J., Kim, C.J., Chen, H.M., Shinn, P., Stevenson, D.K., Zimmerman, J., Barajas, P., Cheuk, R., et al. (2003). Genome-wide insertional mutagenesis of Arabidopsis thaliana. Science 301, 653-657.

Balbi, V., and Devoto, A. (2008). Jasmonate signalling network in Arabidopsis thaliana: crucial regulatory nodes and new physiological scenarios. New Phytol. 177, 301-318.

Baniwal, S.K., Bharti, K., Chan, K.Y., Fauth, M., Ganguli, A., Kotak, S., Mishra, S.K., Nover, L., Port, M., Scharf, K.D., et al. (2004). Heat stress response in plants: a complex game with chaperones and more than twenty heat stress transcription factors. $J$. Biosci. 29, 471-487.

Busch, W., Wunderlich, M., and Schoffl, F. (2005). Identification of novel heat shock factor-dependent genes and biochemical pathways in Arabidopsis thaliana. Plant J. 41, 1-14.

Chen, C.H., and Chen, Z.X. (2002). Potentiation of developmentally regulated plant defense response by AtWRKY18, a pathogeninduced Arabidopsis transcription factor. Plant Physiol. 129, 706-716.

Clarke, S.M., Mur, L.A.J., Wood, J.E., and Scott, I.M. (2004). Salicylic acid dependent signaling promotes basal thermotolerance but is not essential for acquired thermotolerance in Arabidopsis thaliana. Plant J. 38, 432-447.

Clarke, S.M., Cristescu, S.M., Miersch, O., Harren, F.J.M., Wasternack, C., and Mur, L.A.J. (2009). Jasmonates act with salicylic acid to confer basal thermotolerance in Arabidopsis thaliana. New Phytol. 182, 175-187.

Clough, S.J., and Bent, A.F. (1998). Floral dip: a simplified method for Agrobacterium-mediated transformation of Arabidopsis thaliana. Plant J. 16, 735-743.

Dat, J.F., Foyer, C.H., and Scott, I.M. (1998). Changes in salicylic acid and antioxidants during induced thermotolerance in mustard seedlings. Plant Physiol. 118, 1455-1461.

Dat, J.F., Lopez-Delgado, H., Foyer, C.H., and Scott, I.M. (2000). Effects of salicylic acid on oxidative stress and thermotolerance in tobacco. J. Plant Physiol. 156, 659-665.

Dong, J.X., Chen, C.H., and Chen, Z.X. (2003). Expression profiles of the Arabidopsis WRKY gene superfamily during plant defense response. Plant Mol. Biol. 51, 21-37. 
Du, L.Q., and Chen, Z.X. (2000). Identification of genes encoding receptor-like protein kinases as possible targets of pathogenand salicylic acid-induced WRKY DNA-binding proteins in Arabidopsis. Plant J. 24, 837-847.

Eulgem, T., Rushton, P.J., Robatzek, S., and Somssich, I.E. (2000). The WRKY superfamily of plant transcription factors. Trends Plant Sci. 5, 199-206.

Eulgem, T., and Somssich, I.E. (2007). Networks of WRKY transcription factors in defense signaling. Curr. Opin. Plant Biol. 10, 366-371.

Fu, Q.T., Li, S.J., and Yu, D.Q. (2009). Identification of an Arabidopsis nodulin-related protein in heat stress. Mol. Cells 29, 77-84.

Gadjev, I., Vanderauwera, S., Gechev, T.S., Laloi, C., Minkov, I.N., Shulaev, V., Apel, K., Inze, D., Mittler, R., and Van Breusegem, F. (2006). Transcriptomic footprints disclose specificity of reactive oxygen species signaling in Arabidopsis. Plant Physiol. 141, 436-445.

Gong, M., Li, Y.J., Dai, X., Tian, M., and Li, Z.G. (1997). Involvement of calcium and calmodulin in the acquisition of heat-shock induced thermotolerance in maize seedlings. J. Plant Physiol. 150, 615-621.

Guy, C. (1999). The influence of temperature extreme on gene expression, genomic structure, and the evolution of induced tolerance in plants. In Plant responses to environmental stresses, H.R. Lerner, eds. (New York, NY: Marcel Dekker), pp. 497-548.

Hong, S.W., and Vierling, E. (2000). Mutants of Arabidopsis thaliana defective in the acquisition of tolerance to high temperature stress. Proc. Natl. Acad. Sci. USA 97, 4392-4397.

Hong, S.W., Lee, U., and Vierling, E. (2003). Arabidopsis hot mutants define multiple functions required for acclimation to high temperatures. Plant Physiol. 132, 757-767.

Howarth, C.J., Pollock, C.J., and Peacock, J.M. (1997). Development of laboratory-based methods for assessing seedling thermotolerance in pearl millet. New phytol. 137, 129-139.

Howe, G.A. (2004). Jasmonates as signals in the wound response. J. Plant Growth Regul. 23, 223-237.

Jing, S., Zhou, X., Song, Y., and Yu, D. (2009). Heterologous expression of OsWRKY23 gene enhances pathogen defense and darkinduced leaf senescence in Arabidopsis. Plant Growth Regul. 58, 181-190.

Kanna, M., Tamaoki, M., Kubo, A., Nakajima, N., Rakwal, R., Agrawal, G.K., Tamogami, S., loki, M., Ogawa, D., Saji, H., et al. (2003). Isolation of an ozone-sensitive and jasmonate- semiinsensitive Arabidopsis mutant (oji1). Plant Cell Physiol. 44, 1301-1310.

Larkindale, J., and Knight, M.R. (2002). Protection against heat stress-induced oxidative damage in Arabidopsis involves calcium, abscisic acid, ethylene, and salicylic acid. Plant Physiol. $128,682-695$

Larkindale, J., and Huang, B.R. (2005). Effects of abscisic acid, salicylic acid, ethylene and hydrogen peroxide in thermotolerance and recovery for creeping bentgrass. Plant Growth Regul. $47,17-28$.

Larkindale, J., Hall, J.D., Knight, M.R., and Vierling, E. (2005). Heat stress phenotypes of Arabidopsis mutants implicate multiple signaling pathways in the acquisition of thermotolerance. Plant Physiol. 138, 882-897.

Larkindale, J., and Vierling, E. (2008). Core genome responses involved in acclimation to high temperature. Plant Physiol. 146, 748-761.

Li, S.J., Fu, Q.T., Huang, W.D., and Yu, D.Q. (2009). Functional analysis of an Arabidopsis transcription factor WRKY25 in heat stress. Plant Cell Rep. 28, 683-693.

Liu, H.T., Gao, F., Li, G.L., Han, J.L., Liu, D.L., Sun, D.Y., and Zhou, R.G. (2008). The calmodulin-binding protein kinase 3 is part of heat-shock signal transduction in Arabidopsis thaliana. Plant $\mathrm{J}$. $55,760-773$

Lopez-Delgado, H., Dat, J.F., Foyer, C.H., and Scott, I.M. (1998). Induction of thermotolerance in potato microplants by acetylsalicylic acid and $\mathrm{H}_{2} \mathrm{O}_{2}$. J. Exp. Bot. 49, 713-720.
Miller, G., Shulaev, V., and Mittler, R. (2008). Reactive oxygen signaling and abiotic stress. Physiol. Plant 133, 481-489.

Mishra, S.K., Tripp, J., Winkelhaus, S., Tschiersch, B., Theres, K. Nover, L., and Scharf, K.D. (2002). In the complex family of heat stress transcription factors, HSfA1 has a unique role as master regulator of thermotolerance in tomato. Genes Dev. 16, 15551567.

Panchuk, II., Volkov, R.A., and Schoffl, F. (2002). Heat stress- and heat shock transcription factor-dependent expression and activity of ascorbate peroxidase in Arabidopsis. Plant Physiol. 129, 838-853.

Park, C.Y., Lee, J.H., Yoo, J.H., Moon, B.C., Choi, M.S., Kang, Y.H., Lee, S.M., Kim, H.S., Kang, K.Y., Chung, W.S., et al. (2005). WRKY group Ild transcription factors interact with calmodulin. FEBS Lett. 579, 1545-1550.

Pnueli, L., Liang, H., Rozenberg, M., and Mittler, R. (2003). Growth suppression, altered stomatal responses, and augmented induction of heat shock proteins in cytosolic ascorbate peroxidase (Apx1)-deficient Arabidopsis plants. Plant J. 34, 185-201.

Rao, M.V., Lee, H., Creelman, R.A., Mullet, J.E., and Davis, K.R (2000). Jasmonic acid signaling modulates ozone-induced hypersensitive cell death. Plant Cell 12,1633-1646.

Rizhsky, L., Liang, H.J., and Mittler, R. (2002). The combined effect of drought stress and heat shock on gene expression in tobacco. Plant Physiol. 130, 1143-1151.

Sambrook, J., and Russell, D.W. (2001). Molecular cloning: a laboratory manual, (New York: Cold spring harbor laboratory press).

Senaratna, T., Touchell, D., Bunn, E., and Dixon, K. (2000). Acetyl salicylic acid (Aspirin) and salicylic acid induce multiple stress tolerance in bean and tomato plants. Plant Growth Regul. 30, 157-161.

Spoel, S.H., Koornneef, A., Claessens, S.M.C., Korzelius, J.P., Van Pelt, J.A., Mueller, M.J., Buchala, A.J., Metraux, J.P., Brown, R. Kazan, K., et al. (2003). NPR1 modulates cross-talk between salicylate- and jasmonate-dependent defense pathways through a novel function in the cytosol. Plant Cell 15, 760-770.

Suzuki, N., Rizhsky, L., Liang, H.J., Shuman, J., Shulaev, V., and Mittler, R. (2005). Enhanced tolerance to environmental stress in transgenic plants expressing the transcriptional coactivator multiprotein bridging factor 1c. Plant Physiol. 139, 1313-1322.

Suzuki, N., Bajad, S., Shuman, J., Shulaev, V., and Mittler, R. (2008). The transcriptional co-activator MBF1c is a key regulator of thermotolerance in Arabidopsis thaliana. J. Biol. Chem. 283, 9269-9275.

Vijayan, P., Shockey, J., Levesque, C.A., Cook, R.J., and Browse, J. (1998). A role for jasmonate in pathogen defense of Arabidopsis. Proc. Natl. Acad. Sci. USA 95, 7209-7214.

von Koskull-Döring, P., Scharf, K.D., and Nover, L. (2007). The diversity of plant heat stress transcription factors. Trends Plant Sci. 12, 452-457.

Wasternack, C. (2006). Oxylipins: biosynthesis, signal transduction and action. In Plant Hormone Signaling, P. Hedden, and S. Thomas, eds., (Oxford, UK: Blackwell publishing Ltd.), pp. 185228.

Wasternack, C. (2007). Jasmonates: an update on biosynthesis, signal transduction and action in plant stress response, growth and development. Ann. Bot. 100, 681-697.

Weigel, D., and Glazebrook, J. (2002). Arabidopsis: a laboratory manual, (New York: Cold Spring Harbor Laboratory Press).

Yu, D.Q., Chen, C.H., and Chen, Z.X. (2001). Evidence for an important role of WRKY DNA binding proteins in the regulation of NPR1 gene expression. Plant Cell 13,1527-1539.

Zheng, Z.Y., Mosher, S.L., Fan, B.F., Klessig, D.F., and Chen, Z.X. (2007). Functional analysis of Arabidopsis WRKY25 transcription factor in plant defense against Pseudomonas syringae. BMC Plant Biol. 7, 13.

Zhang, W., Zhou, R.G., Gao, Y.J., Zheng, S.Z., Xu, P., Zhang, S.Q., and Sun, D.Y. (2009). Molecular and genetic evidence for the key role of AtCaM3 in heat-Shock signal transduction in Arabidopsis. Plant Physiol. 149, 1773-1784. 